Correspondence for

Frantz, S. UK trial disaster could spell trouble for industry. Nature Rev. Drug Discov. 5, 364-365 (2006).

\title{
Minimizing disasters during early drug development: A pressing need for an improved guidance
}

\author{
Bernd Jilma* and Rashmikant Shah**
}

*Dept. of Clinical Pharmacology, Division of I mmunology and Haematology, Medical University of Vienna, Vienna, Austria. **Rashmi Shah Pharmaceutical Services, Bucks, UK. e-mails: Bernd.J ilma@meduniwien.ac.at; clin.safety@ineone.net

Clinical trials with new investigational medicinal products necessarily have to go through a number of phases, usually beginning with healthy volunteers, in order to progressively establish their safety and ultimately their efficacy. Recently, a contract research organization was conducting the first human testing of TGN1412 (TeGenero AG), an immunomodulatory humanized agonistic anti-CD28 monoclonal antibody (MAb) ${ }^{1.2}$. Six healthy volunteers received an infusion of the anti-CD28 antibody TGN1412, and two volunteers were administered placebo. All the six volunteers receiving TGN1412 fell critically ill with potentially life-threatening complications within hours of starting drug infusion.

Disasters occur only rarely during Phase I trials, which have a remarkable record of safety, and should always elicit our immediate attention to further improve the safety of this early phase clinical drug development.

First, the pharmacology of a drug often provides a clue to the potential toxicity that one might encounter. Conventional anti-CD28 MAbs are, only in the context of costimulation, capable of driving interleukin- 2 production and T-cell proliferation ${ }^{3}$. In contrast, superagonistic anti-CD28 antibodies do not depend on exogenous triggers of T cell receptor to initiate full T cell activation ${ }^{4}$. Notably, interleukin- 2 is also known to be a drug with narrow therapeutic index as it sometimes induces life-threatening capillary leak syndrome ${ }^{5}$. This can result in fulminant respiratory and renal insufficiency. Against the background of this knowledge, alarm bells should toll particularly because the investigational antibody is activating rather than inhibiting $T$ cells. Second, even toxicology studies in higher animals such as monkeys may provide a false sense of security and apparent safety if there is little inter-species cross-reactivity for an antibody or if the response pattern may be highly specific for humans. This is also reflected in the paragraph on "Animal models with limited utility" of a recent FDA guidance document ${ }^{6}$. These issues are not as trivial, as performing a drug interaction study or another bioequivalence study, and may be more apparent to investigators experienced in Phase I trials with pro-inflammatory agents. One needs to consider whether this disaster is a timely pointer to consider restricting Phase I studies with such novel drugs to specialized centers. Clinical pharmacologists with a strong scientific background in the particular field of science should play a key role as investigators ${ }^{7}$. The expertise available in spezialized centres could help to identify early any potential or theoretical risks, improve 
designs of clinical trial protocols, address other possible shortcomings in the scientific rationale that support the safety of similar agents.

The most striking feature of the study with TGN1412 was that six volunteers were exposed simultaneously to a drug with an unknown safety profile in humans. This is counter-intuitive particularly in a first-in-man dose escalation trial. Exposing only 1 or 2 subjects to start with on a single day, particularly at the start of each dose level, would generate significant insights into safety. This approach, although time-consuming, would result in a substantial reduction in exposure of subjects to potentially unknown hazards associated with such novel drugs. However, the approach selected is often related to expediency, economics and logistics and usually not regulated by regulatory, medical, ethical or clinical research guidelines. This disaster, is also a timely warning that calls for more stringent regulation and standard operating procedures governing first-in-man trials. These should not only provide improved recommendations on doses but also limit the number of subjects who are simultaneously exposed to a drug whose safety in humans is unknown.

1. TeGenero, Statement über TGN1412. [online], <http://www.tegenero.de/news/statement ueber tgn1412/index.php> (2006).

2. Frantz, S. UK trial disaster could spell trouble for industry. Nature Rev. Drug Discov. 5, 364-365 (2006).

3. Beyersdorf, N., Hanke, T., Kerkau, T. \& Hunig T. Superagonistic anti-CD28 antibodies: potent activators of regulatory T cells for the therapy of autoimmune diseases Ann. Rheum. Dis.. 64, S4, iv91-95 (2005).

4. Riley, J. L, \& J une C. H. The CD28 family: a T-cell rheostat for therapeutic control of T-cell activation. Blood 105, 13-21 (2005).

5. Rosenberg, S. A. et al. Observations on the systemic administration of autologous lymphokine-activated killer cells and recombinant interleukin-2 to patients with metastatic cancer. N. Engl. J. Med. 313, 1485-1492 (1985).

6. FDA. Guidance for industry estimating the maximum safe starting dose in initial clinical trials for therapeutics in adult healthy volunteers. [online], $<$ www.fda.gov/cder/guidance/5541fnl.htm>(2005).

7. Maxwell, S. R. \& Webb, D.J . Clinical pharmacology - too young to die? Lancet 367, 799-800 (2006). 